\title{
Line Flow Based WLAV State Estimation Using PSO Technique
}

\author{
M.Kalpanadevi ${ }^{1}$, R.Neela ${ }^{2}$ \\ ${ }^{1}$ (Assistant Professor, Dept. of Electrical Engg., Annamalai University, India) \\ ${ }^{2}$ (Professor, Dept. of Electrical Engg., Annamalai University, India)
}

\begin{abstract}
State Estimation techniques are widely used to estimate the operating state of power systems in the most reliable manner so that the estimated state variables reflect the current system state faithfully and these studies enable the energy management centre to operate the system in secure manner under normal as well as contingent conditions. Those algorithms which generate a fairly good estimate in spite of the presence of bad measurements are considered to be comparatively superior and WLAV based SE has been widely accepted as one such technique whose outcome is almost immune to the existence of bad measurements. In this paper, a new, line flow based WLAV state estimation (WLAV-LFBSE) technique for power systems using line flow measurements, bus power injections and bus voltage magnitudes as measurement vectors has been suggested and it has been solved through PSO technique. The proposed method has been tested on standard test systems taking into account various percentages of bad measurements and the results are analyzed.
\end{abstract}

Keywords - State Estimation, Weighted Least Absolute value method, Line flow based WLAV, LFWLAV-PSO and Power System.

\section{Introduction}

State estimation techniques were developed for the purpose of generating a data base for power system studies from the available redundant set of erroneous measurements. In the present day circumstances where the power systems are operated under constantly growing stress, system security is under great threat and to ensure that the system operates in a secured manner even under contingent conditions, the system is to be monitored continuously and corrective actions are to be implemented to limit the out of bound voltages and to relieve the lines off their overloads. This makes it inevitable to use real time monitoring and estimating techniques that are fast and reliable. WLS technique has been widely used for solving the problem of state estimation. (1). Many variants of this WLS technique are available, each one thriving in its own way to generate a convincing estimate within a reasonable time. Though computationally simple, WLS based techniques are prone to the ill effects caused by the presence of bad measurements in the measurement vector and the focus has shifted towards obtaining a good estimate even in the presence of such bad measurements.

A SE algorithm, based on weighted least absolute value (WLAV) minimization technique, has been alternatively used to handle power system problems [2-3]. Unlike WLS method, there is no explicit formula for the solution of WLAV algorithm but it can be reformulated as a linear programming (LP) problem. The estimate is then obtained by solving a sequence of LP problems. It is well known that this estimator is capable of automatically rejecting bad data, as long as the bad measurements are not leverage points, and hence found to be more robust than a WLS estimator [4].The need for an efficient algorithm that occupies minimum memory and requires lower computation time has led to the development of fast decoupled state estimation (FDSE) [5-10] based on $P-\delta$ and $Q-V$ natural decoupling. The rate of convergence is strongly influenced by the initial voltages, which sometimes have a large $\delta$ and a poor $V$ and the coupling between $P-\delta$ and $Q-V$ mathematical models.

The line flow and bus voltage magnitude load flow model presented in (11) has been used in this paper to develop a similar SE model which has been solved using WLS technique by applying PSO algorithm. This approach tends to avoid most of the factorization related matrix manipulation problems mentioned so far.

Presence of bad data has a substantial impact over the quality of the estimate generated by the least squares based estimator and hence special techniques were needed to identify them and quantify their effects. A linear recursive bad data identification technique based on power system decomposition has been presented in (12). Neural network based filter has been applied for bad data detection and identification in(13) in which once trained, the filter quickly identifies most measurement errors simultaneously by comparing the square difference of raw measurements and their corresponding estimated values with some given thresholds. Bad data prefiltering using wavelet transform has been presented in (14) and this approach identifies and filters out the bad data even before the state estimation algorithm estimates the system state. An identification algorithm based on the largest normalized residual considering statistical correlation among the measurements is presented in (15). As the proposed method here uses a constant Jacobian, unlike the conventional WLS estimator, the impact 
of bad measurements over the estimate has been considerably reduced and hence it doesn't require a separate algorithm to filter out the bad measurements. Over the past few decades soft computing algorithms have been playing a major role in solving optimization problems. Evolutionary programming algorithms are promising from the point of view of their capability to evade local maxima and minima. Out of the many evolutionary algorithms PSO has been widely used from the point of view of assured convergence and programming flexibility. PSO algorithm has been successfully implemented for solving the problem of SE inspite of the apprehensions such as larger computational time etc (16). In this work a line flow based WLAV state estimation problem has been formulated and it has been solved through PSO technique in the absence as well as the presence of bad measurements for various standard IEEE test systems.

\subsection{Conventional WLAV State Estimation}

\section{Problem formulation}

The WLS estimator is not a robust one because of its quadratic objective function. Therefore, an estimator involving non-quadratic objective function is used. This estimator offers a more robust estimation, which is obtained by minimising

$$
\begin{aligned}
& J=\left[\operatorname{diag}\left(R^{-1}\right)\right]^{T}|z-h(x)| \\
& =\sum_{j=1}^{n z}\left|z_{j}-h_{j}(x)\right| / \sigma_{j}{ }^{2}
\end{aligned}
$$

Since the above objective minimises the absolute value of the error weighted by the measurement accuracy $\sigma_{j}{ }^{-2}$, it is commonly called as the WLAV estimator.

The objective of Eq. (1) is reformulated using LP in order to solve the WLAV problem:

Minimise $J=\left[\operatorname{diag}\left(R^{-1}\right)\right]^{T}[\gamma+\eta]$

Subject to

$H \Delta x+\gamma-\eta=\Delta z$

$\gamma, \eta \geq 0$

A SE solution is obtained by solving the LP problem given by Eq. (3) iteratively for $x$ until $\Delta x$ is sufficiently small. This method is highly inefficient, as it requires large computer memory and involves the time consuming LP technique, which itself is an iterative process and hence not suitable for real time applications. However this algorithm is robust and stable in the sense that it has the inherent feature of rejecting bad measurements by interpolating only $n s$ among the $n z$ measurements and free from ill-conditioning due to the effect of wide assignment of weighting factors and avoidance of factorisation and multiplication of several matrices. In this paper an attempt has been made to increase the computational efficiency of the robust WLAV technique through linearization.

\subsection{Proposed Method}

The real and reactive bus powers as a function of real line flows, reactive line flows and $\mathrm{V}_{\mathrm{m}}{ }^{2}$ can be written as

$$
\begin{aligned}
& P_{i}=\sum_{j=1}^{n l} A_{i j} p_{j}-\sum_{j=1}^{n l} A_{i j}^{\prime} l_{j} \\
& Q_{i}=\sum_{j=1}^{n l} A_{i j} q_{j}-\sum_{j=1}^{n l} A_{i j} m_{j}
\end{aligned}
$$

Treating $\mathrm{P}, \mathrm{Q}$ and $\mathrm{V}_{\mathrm{m}}{ }^{2}$ as state variable[x], the measurement set $[\mathrm{Z}]$ can be represented as

$[Z]=[f(x)]$

Where

$[Z]=\left[P, Q, p, q, V^{2}\right]^{T}$

The WLAV objective function can be written as

$\operatorname{Min} \varphi=\sum_{i=1}^{n m} w i[Z i-f i(x)]$

The above equation does not include line capacitances and shunt susceptances and hence it is inadequate to estimate the system state. However the problem can be made solvable if constraint equations including branch voltage drop and phase angle drop are considered. These constraints can be represented as

$h(x)=2 R p+2 X q-\left(\Lambda A_{1+}^{T}+A_{1-}^{T}\right) V^{2}=0$ 
$g(x)=C X p-C R q-C \alpha=0$

The constrained optimization problem of equations 7,8 and 9 can be formulated as a linear programming problem as

$\operatorname{Min} \varphi=\sum_{i=1}^{n m} w i\left[S i^{\prime}-S i^{\prime \prime}\right]$

Subject to

A. $\Delta x+S^{\prime}-S^{\prime \prime}=Z-f\left(x^{0}\right)$

H. $\Delta x=-h\left(x^{0}\right)$

G. $\Delta x=-\mathrm{g}\left(\mathrm{x}^{0}\right)$

Where

$A, H$ and $G$ are the jacobian matrices formed by partially differenting $f(x), h(x)$ and $g(x)$ with respect to $x$.

$\Delta \mathrm{x}$ is the state correction vector

$\mathrm{S}^{\prime}$ and $\mathrm{S}$ " are the slack variable vectors.

The above LP problem can be solved iteratively for $\mathrm{x}$ till the algorithm converges. It is to be noted that the jacobian matrices $\mathrm{A}, \mathrm{H}$ and $\mathrm{G}$ are constant matrices that require to be computed only at the beginning of the iterative process. However RHS vectors $\mathrm{f}(\mathrm{x}), \mathrm{g}(\mathrm{x}) \mathrm{h}(\mathrm{x})$ must be recomputed during iterative process.

\subsubsection{Introduction of PSO}

PSO was first introduced by Kennedy and Eberhart in 1995 and it is a heuristic optimization technique induced by the swarm intelligences of animals such as bird flocking, fish schooling. A swarm of particles represent a solution to the optimization problem. Each particle adjusts its position according to its own experience and the experience of its neighboring particles. The position and velocity of $i^{\text {th }}$ particle in an $\mathrm{N}-$ dimensional search space is represented as

$X_{i}=\left(x_{i 1}, x_{i 2}, \ldots \ldots x_{i n}\right)$

$v_{i}=\left(v_{i 1}, v_{i 2}, \ldots \ldots v_{\text {in }}\right)$

The best position achieved by a particle is recorded and is denoted by

$P_{\text {best } i}=\left(x_{i 1}^{P_{\text {best }}}, \ldots \ldots x_{\text {in }}^{P_{\text {best }}}\right)$

The best particle among all the particles in the population is represented by

$G_{\text {best } i}=\left(x_{i 1}^{G_{\text {best }}}, \ldots \ldots x_{\text {in }}^{G_{\text {best }}}\right)$

The updated velocity and position of each particle in $(k+1)^{t h}$ step are calculated as follows

$X_{i}^{k+1}=X_{i}^{k}+V_{i}^{k+1}$

Where,

$V_{i}{ }^{k+1}=w V_{i}^{k}+C_{1}$ rand $_{1}\left(x_{i} P_{\text {best } i}^{k}-x_{i}^{k}\right)+C_{2}$ rand $_{2}\left(G P_{\text {best } i}^{k}-x_{i}^{k}\right)$

where

$$
\begin{gathered}
C 1=C_{1} \max -\left\{\frac{C_{1 \max }-C_{1 \min }}{\text { Iter }_{\text {max }}}\right\} x \text { Iter } \\
C 2=C_{2} \max -\left\{\frac{C_{2 \max }-C_{2 \min }}{\text { Iter }_{\text {max }}}\right\} x \text { Iter } \\
w=w_{\text {max }}-\left\{\frac{w_{\text {max }}-w_{\text {min }}}{\text { Iter } r_{\max }}\right\} x \text { Iter }
\end{gathered}
$$

In this velocity updating process, the acceleration coefficients $C_{1}, C_{2}$ and weight parameter ' $w$ ' are predefined and $\operatorname{rand}_{1}$ andrand $\mathrm{are}_{2}$ uniformly generated random numbers in the range of $[0,1]$ and this velocity updation is carried out until stopping criterion is reached/met.

\subsubsection{PSO Algorithm}

1. Choose the population size, the number of generations, $\mathrm{W}_{\min }, \mathrm{W}_{\max }, \mathrm{C}_{1 \min }, \mathrm{C} 1 \max , \mathrm{C} 2 \mathrm{~min}, \mathrm{C} 2 \mathrm{max}$, pbest, gbest.

2. Initialize the velocity and position of all particles randomly, ensuring that they are within limits. Here the individuals represent the real and reactive power flows and bus voltage magnitudes.

3. Set the generation counter $\mathrm{t}=1$.

4. Evaluate the fitness for each particle using equation (10) according to the objective function.

5. Compare the particle's fitness function with its $P_{\text {best } i}$. If the current value is better than

$P_{\text {best } i}$, then set $P_{\text {best } i}$ is equal to the current value. Identify the particle in the neighborhood with the best success so far and assign it to Gbest. 
6. Update velocity by using the global best and individual best of the particle.

7. Update position by using the updated velocities. Each particle will change its position.

8. If the stopping criteria is not satisfied set $t=t+1$ and go to step 4.Otherwise stop.

\section{III. simulation and results}

The proposed LFBSE problem has been solved using PSO technique by selecting a population size of 20 and generation size of 50 and it has been tested on standard IEEE 14, 30 and 57 bus test systems. The measurement vector has been generated by adding a small percentage of noise to the values obtained from the Newton Raphson load flow. Bus voltage magnitudes at the load buses and real and reactive power flows through the lines were taken as state variables. All the line flows, bus power injections and bus voltage magnitudes at the even numbered buses were considered in the measurement set to achieve necessary redundancy. To study the performance of the algorithm in the presence as well as absence bad measurements, in each of the measurement set, 5, 10 and 15 number of bad measurements were introduced randomly. The performance of the algorithm has been validated by comparing the results of the proposed method against the results obtained using standard WLAV state estimation and LFWLAV State Estimation The algorithms were tested with a flat start and a convergence tolerance of 0.0001 . Three performance indices are defined to validate the performance of the proposed technique. They are $\Delta \mathrm{V}_{\mathrm{rms}}, \Delta \mathrm{p}_{\mathrm{rms}}, \Delta \mathrm{q}_{\mathrm{rms}}$.

$\begin{aligned} \Delta V r m s & =\sqrt{\frac{1}{n b}} \sum_{i}^{n b}\left(V_{i}^{t}-V_{i}\right)^{2} \\ \Delta p_{r m s} & =\sqrt{\frac{1}{n l}} \sum_{i}^{n l}\left(P_{i}^{t}-P_{i}\right)^{2} \\ \Delta q_{\text {rms }} & =\sqrt{\frac{1}{n l}} \sum_{i}^{n l}\left(q_{i}^{t}-q_{i}\right)^{2}\end{aligned}$

Tables 1, 2 and 3 compare the performance of the proposed method with WLAV and LFWLAV estimation algorithm in terms of the performance indices defined in 1,2 and 3 and NET. The performance of the algorithm is also illustrated through bar charts in Fig 1 to 12.

Table 1: Results for IEEE 14 Bus Systems

\begin{tabular}{|c|c|c|c|c|c|}
\hline Measurements & Method & $\Delta \mathrm{Vrms}$ & $\Delta$ Prms & $\Delta$ Qrms & NET in $m s$ \\
\hline \multirow[t]{3}{*}{0} & WLAV & 0.1406 & 0.1351 & 0.1643 & 211 \\
\hline & LFWLAV & 0.0883 & 0.1103 & 0.111 & 136 \\
\hline & LFWLAV-PSO & 0.0872 & 0.1097 & 0.1097 & 143 \\
\hline \multirow[t]{3}{*}{5} & WLAV & 0.1405 & 0.1286 & 0.1631 & 210 \\
\hline & LFWLAV & 0.0882 & 0.1074 & 0.1094 & 136 \\
\hline & LFWLAV-PSO & 0.0867 & 0.1045 & 0.1085 & 145 \\
\hline \multirow[t]{3}{*}{10} & WLAV & 0.1363 & 0.1277 & 0.1573 & 212 \\
\hline & LFWLAV & 0.0635 & 0.1034 & 0.1083 & 137 \\
\hline & LFWLAV-PSO & 0.0585 & 0.1033 & 0.1079 & 146 \\
\hline \multirow[t]{3}{*}{15} & WLAV & 0.1349 & 0.1215 & 0.138 & 212 \\
\hline & LFWLAV & 0.0246 & 0.1027 & 0.1078 & 137 \\
\hline & LFWLAV-PSO & 0.0233 & 0.1026 & 0.107 & 146 \\
\hline
\end{tabular}

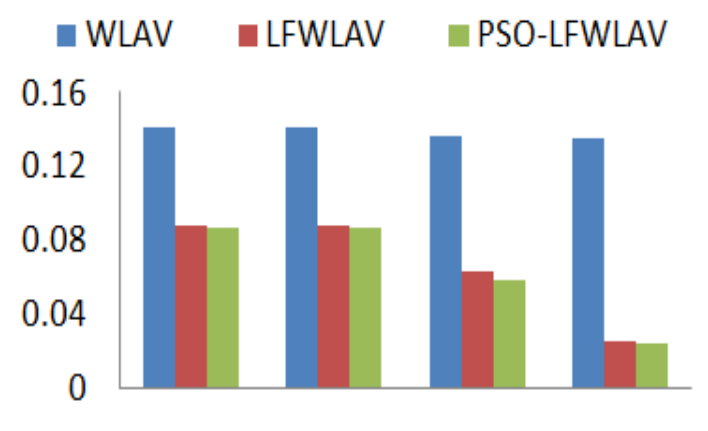

0

5

10

15

Fig.1: Measurement Vs $\Delta$ Vrms (14Bus)

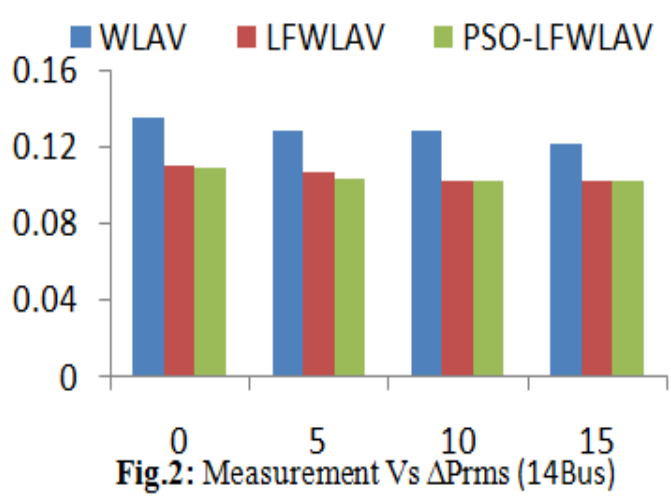

Fig.2: Measurement Vs $\triangle$ Prms (14Bus) 


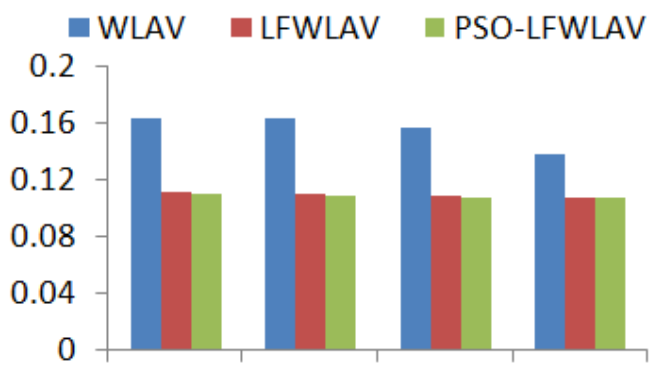

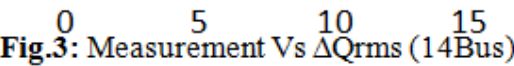

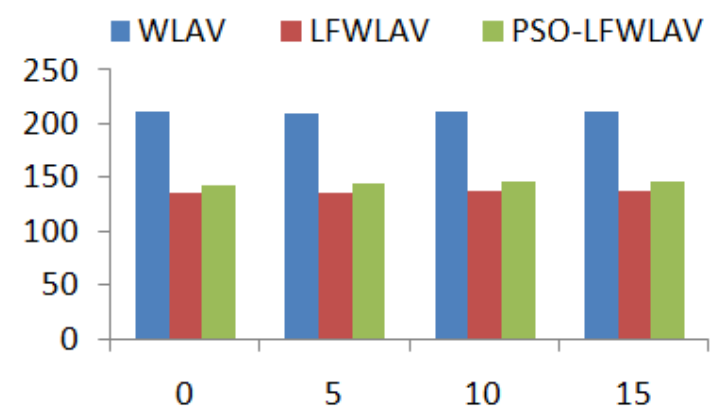

Fig.4: Measurement Vs NET (14Bus)

Table 2: Results for IEEE 30 Bus Systems

\begin{tabular}{|c|c|c|c|c|c|}
\hline Measurements & Method & $\Delta$ Vrms & $\Delta$ Prms & $\Delta$ Qrms & NET in ms \\
\hline \multirow{4}{*}{0} & WLAV & 0.1742 & 0.3824 & 0.2117 & 468 \\
\cline { 2 - 6 } & LFWLAV & 0.0755 & 0.2173 & 0.1325 & 189 \\
\cline { 2 - 6 } & LFWLAV-PSO & 0.0659 & 0.2165 & 0.1318 & 197 \\
\hline \multirow{3}{*}{5} & WLAV & 0.0833 & 0.3794 & 0.2109 & 469 \\
\cline { 2 - 6 } & LFWLAV & 0.0397 & 0.2159 & 0.1319 & 188 \\
\cline { 2 - 6 } & LFWLAV-PSO & 0.0314 & 0.2157 & 0.1314 & 197 \\
\hline \multirow{3}{*}{10} & WLAV & 0.0609 & 0.3756 & 0.2099 & 469 \\
\cline { 2 - 6 } & LFWLAV & 0.0328 & 0.2138 & 0.1311 & 189 \\
\cline { 2 - 6 } & LFWLAV-PSO & 0.0312 & 0.213 & 0.1307 & 199 \\
\cline { 2 - 6 } & WLAV & 0.0454 & 0.3743 & 0.2081 & 469 \\
\cline { 2 - 6 } & LFWLAV & 0.0283 & 0.213 & 0.1305 & 189 \\
\cline { 2 - 6 } & LFWLAV-PSO & 0.0244 & 0.2024 & 0.1299 & 200 \\
\hline
\end{tabular}

- WLAV " LFWLAV " PSO-LFWLAV

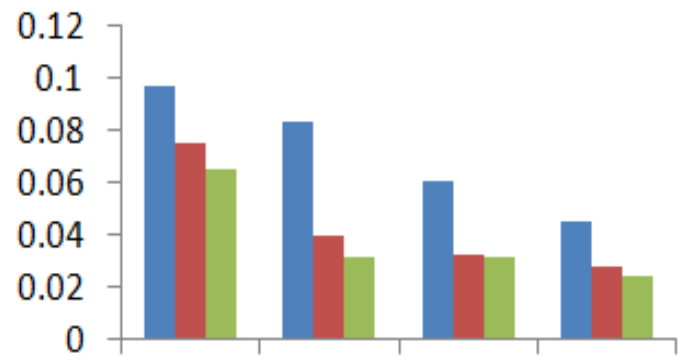

$\begin{array}{llll}0 & 5 & 10 & 15\end{array}$

Fig.5: Measurement Vs $\Delta \mathrm{Vrms}$ (30Bus)

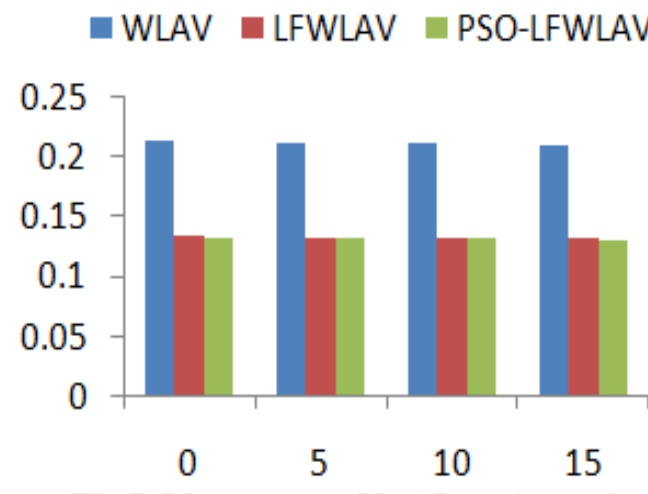

Fig.7: Measurement Vs $\Delta$ Qrms (30Bus)

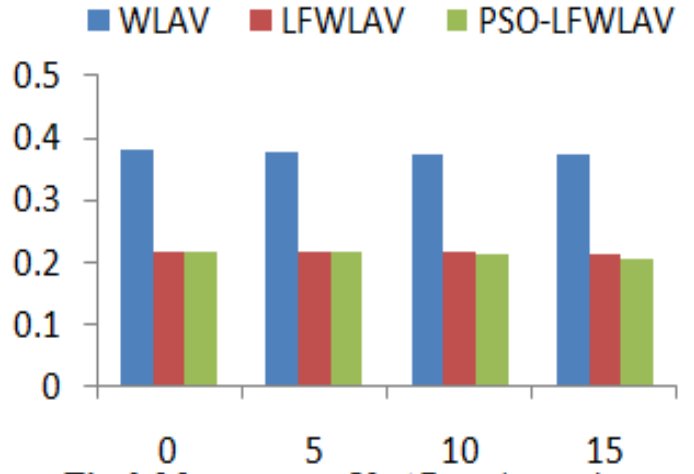

Fig.6: Measurement Vs $\Delta$ Prms (30Bus)

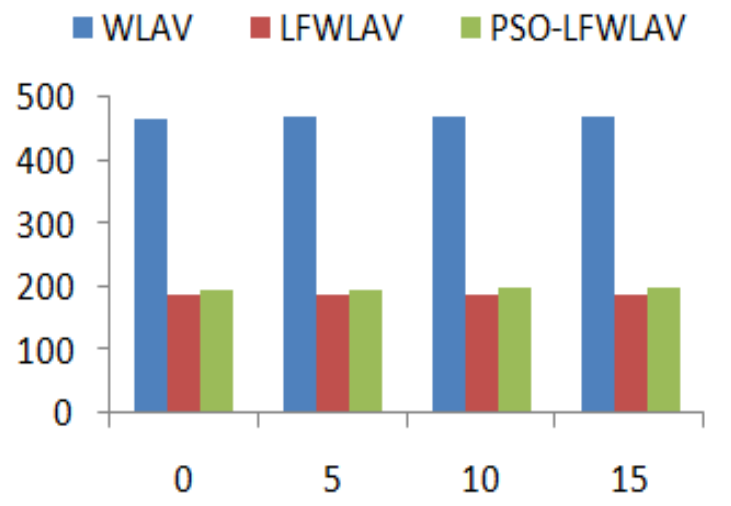

Fig.8: Measurement Vs NET (30Bus) 
Table 3: Results for IEEE 57 Bus Systems

\begin{tabular}{|c|c|c|c|c|c|}
\hline Measurements & Method & $\Delta$ Vrms & $\Delta$ Prms & $\Delta$ Qrms & NET in ms \\
\hline \multirow{4}{*}{0} & WLAV & 0.0791 & 0.2579 & 0.1346 & 711 \\
\cline { 2 - 6 } & LFWLAV & 0.0288 & 0.1173 & 0.1091 & 233 \\
\cline { 2 - 6 } & LFWLAV-PSO & 0.0287 & 0.1161 & 0.1083 & 249 \\
\hline \multirow{3}{*}{5} & WLAV & 0.0788 & 0.2553 & 0.1332 & 709 \\
\cline { 2 - 6 } & LFWLAV & 0.0283 & 0.1164 & 0.1083 & 234 \\
\cline { 2 - 6 } & LFWLAV-PSO & 0.0269 & 0.1152 & 0.1075 & 249 \\
\cline { 2 - 6 } & WLAV & 0.0782 & 0.2527 & 0.132 & 709 \\
\cline { 2 - 6 } & LFWLAV & 0.0272 & 0.1158 & 0.1071 & 232 \\
\hline \multirow{3}{*}{10} & LFWLAV-PSO & 0.0251 & 0.114 & 0.1052 & 250 \\
\cline { 2 - 6 } & WLAV & 0.0774 & 0.2502 & 0.1313 & 710 \\
\cline { 2 - 6 } & LFWLAVAV & 0.0258 & 0.115 & 0.106 & 232 \\
\hline
\end{tabular}

WLAV — LFWLV — PSO-LFWLAV

WLAV IFWLAV IPSO-LFWLAV

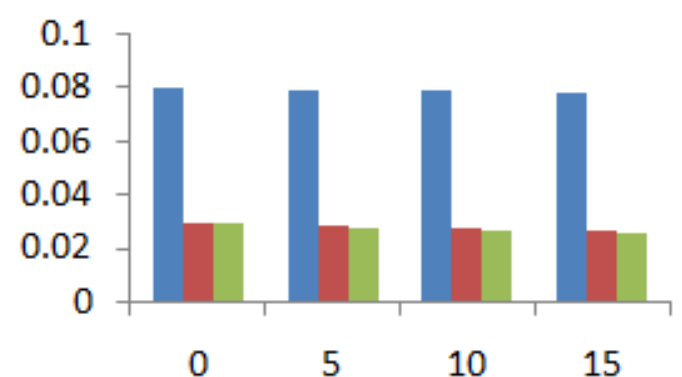

Fig.9: Measurement Vs $\Delta$ Vrms (57Bus)

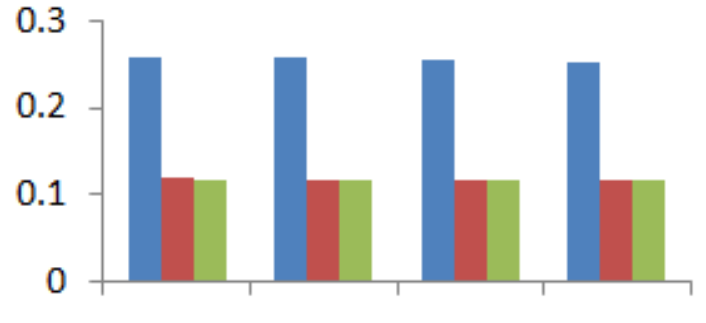

$\begin{array}{llll}0 & 5 & 10 & 15\end{array}$

Fig.10: Measurement Vs $\Delta$ Prms (57Bus)
"WLAV $\square$ LFWLAV $\square$ PSO-LFWLAV

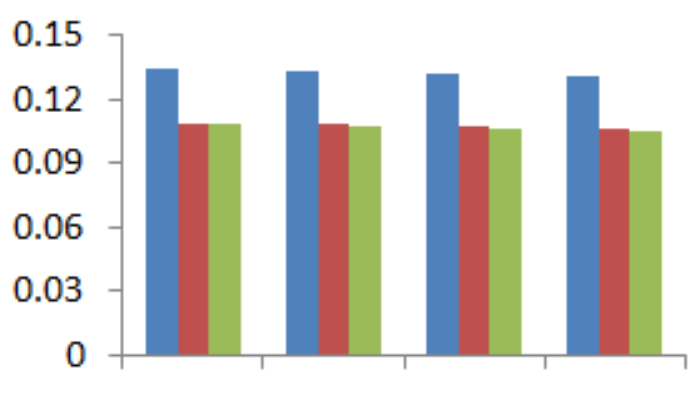

$\begin{array}{llll}0 & 5 & 10 & 15\end{array}$

Fig.11: Measurement Vs $\Delta$ Qrms (57Bus)
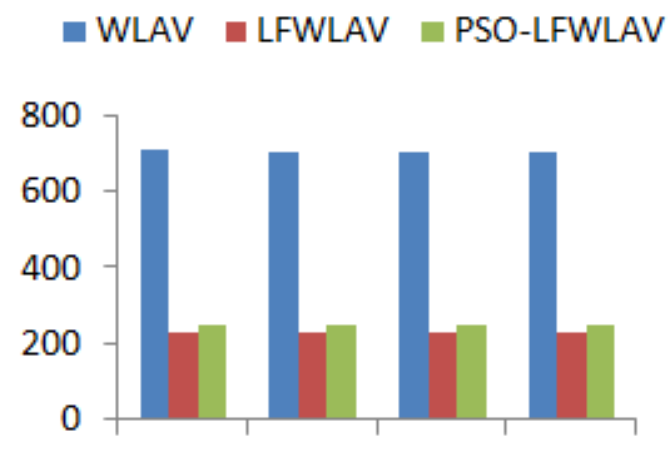

$\begin{array}{llll}0 & 5 & 10 & 15\end{array}$

Fig.12: Measurement Vs NET (57Bus)

\section{Conclusion}

A novel line flow based state estimation technique which results in the formation of constant jacobian matrix has been presented in this paper and it has been solved through WLAV method. PSO technique has been implemented for solving the LFBWLAV problem in the presence as well as absence of bad measurements. The results indicate that the normalized value of the error between the actual values and estimated values of the state variables is considerably lesser in the case of proposed method when solved using PSO than that of the conventional WLAV and LFBWLAV techniques. There is a marginal increase in computation time due to the heuristic search nature of PSO algorithms still as the estimated system state is more closer to the actual system state in the proposed method, this method is highly suitable for security studies of power systems.

\section{References}

[1]. Schweppe. F. C. and Wildes. J., "Power System Static State estimation", Part I: Exact Model, IEEE Tras. Power Appar.Syst., 1978,Vol. PAS-89, pp. 120-125.

[2]. M.R. Irving. R.C. Owen and M.J.H. Sterling, "Power system state estimation using linear programming", Proceedings of IEE. 1978, Vol. 125, pp. 879-885. 
[3]. W.W. Kotiuga and M.Vidyasagar, "Bad data rejection properties of weighted least absolute techniques applied to static state estimation", IEEE Trans. on Power Appar. and Syst., 1982, Vol. PAS-101, No.4, pp.844-853.

[4]. Ali Abur. "A bad data identification method for linear programming for state estimation", IEEE Trans. on Power Systems, 1990, Vol. 5, No. 3, pp. 894-901.

[5]. H.P. Horisberger. J.C. Richard and C. Rossier, "A fast decoupled static state-estimator for electric power systems", IEEE Trans. on Power Appar. and Syst., 1976, Vol. PAS-95, No.1, pp.208-215.

[6]. N.D. Rao and S.C. Tripathy, "A variable step size decoupled state estimator", IEEE Trans. on Power Appar. and Syst., 1979 , Vol. PAS-98, No. 2, pp. 436-443.

[7]. J.J. Allemong. L. Radu and A.M. Sasson, "A fast and reliable state estimation algorithm for AEP's new control centre", IEEE Trans. on Power Appar. and Syst., .1982, Vol. PAS-101, No. 4, pp. 933-944.

[8]. W.M. Lin and J.H. Teng., "Distribution fast decoupled state estimation by measurement pairing", IEE Proc. Gener. Transm. Distrib., 1996, Vol. 143, No. 1, pp. 43-48.

[9]. W.M. Lin and J.H. Teng., "A new transmission fast-decoupled state estimation with equality constraints", International Journal of Electrical Power and Energy Systems, 1998, Vol. 20, No. 7, pp. 489-493.

[10]. L. Roy and T.A. Mohammed, "Fast super decoupled state estimator for power systems", IEEE Trans. on Power Systems, 1997 , Vol. 12, No. 4, pp. 1597-1603.

[11]. P.Yan, A.Sekar, "Study state analysis of power system having multiple Facts devices using line flow based equations", IET Proceedings-Generation Transmission and Distribution, 2005, Vol.152, Issue 1, pp.31-39.

[12]. B.M.Zhang, S.Y.Wang and N.D.Xiang, "A linear recursive bad data identification method with real time application to power system state estimation”, IEEE Trans. on Power Systems, 1992, Vol.7, No.3, pp.1378-1385. 